INPLASY

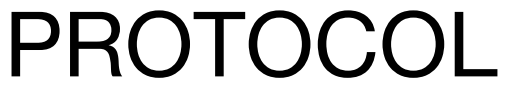

To cite: Wang et al. A systematic review and network meta-analysis: Role of SNPs in predicting breast carcinoma risk. Inplasy protocol 202220092. doi:

10.37766/inplasy2022.2.0092

Received: 21 February 2022

Published: 22 February 2022

Corresponding author:

Yingying Zhang

csuzhangyingying@163.com

Author Affiliation:

Institutional address:

Department of Oncology,

Xiangya Hospital of Central

South University, Changsha, People's Republic of China, 410078.

Support: Not applicable.

Review Stage at time of this submission: Data analysis.

Conflicts of interest:

None declared.

\section{A systematic review and network meta-analysis: Role of SNPs in predicting breast carcinoma risk}

Review question / Objective: P: Breast cancer patient; I: Single nucleotide polymorphisms associated with breast cancer risk; C: Healthy person; O: By comparing the proportion of SNP mutations in the tumor group and the control group, the effect of BREAST cancer risk-related SNP was investigated; S: Case-control study.

Condition being studied: Breast cancer (BC) is one of the most common cancers among women, and its morbidity and mortality have continued to increase worldwide in recent years, reflecting the strong invasiveness and metastasis characteristics of this cancer. BC is a complex disease that involves a sequence of genetic, epigenetic, and phenotypic changes. Polymorphisms of genes involved in multiple biological pathways have been identified as potential risks of BC. These genetic polymorphisms further lead to differences in disease susceptibility and severity among individuals. The development of accurate molecular diagnoses and biological indicators of prognosis are crucial for individualized and precise treatment of $\mathrm{BC}$ patients.

INPLASY registration number: This protocol was registered with the International Platform of Registered Systematic Review and Meta-Analysis Protocols (INPLASY) on 22 February 2022 and was last updated on 22 February 2022 (registration number INPLASY202220092).

\section{INTRODUCTION}

Review question / Objective: P: Breast cancer patient; I: Single nucleotide polymorphisms associated with breast cancer risk; C: Healthy person; O: By comparing the proportion of SNP mutations in the tumor group and the control group, the effect of BREAST cancer risk-related SNP was investigated; S: Casecontrol study.

Condition being studied: Breast cancer (BC) is one of the most common cancers 
among women, and its morbidity and mortality have continued to increase worldwide in recent years, reflecting the strong invasiveness and metastasis characteristics of this cancer. BC is a complex disease that involves a sequence of genetic, epigenetic, and phenotypic changes. Polymorphisms of genes involved in multiple biological pathways have been identified as potential risks of BC. These genetic polymorphisms further lead to differences in disease susceptibility and severity among individuals. The development of accurate molecular diagnoses and biological indicators of prognosis are crucial for individualized and precise treatment of $\mathrm{BC}$ patients.

\section{METHODS}

Search strategy: $((()(((" P o l y m o r p h i s m$, Single Nucleotide"[Mesh]) OR (Nucleotide Polymorphism, Single[Title/Abstract])) OR (Nucleotide Polymorphisms, Single[Title/ Abstract])) OR (Polymorphisms, Single Nucleotide[Title/Abstract])) OR (Single Nucleotide Polymorphisms[Title/Abstract])) OR (SNPs[Title/Abstract])) OR (Single Nucleotide Polymorphism[Title/Abstract])) OR (rs[Title/Abstract])) AND (("Breast Neoplas m s " [Mesh]) OR

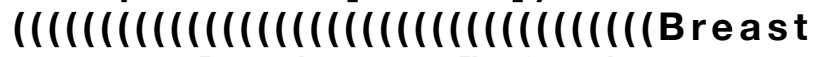
Neoplasm[Title/Abstract]) OR (Neoplasm, Breast[Title/Abstract])) OR (Breast Tumors[Title/Abstract])) OR (Breast Tumor[Title/Abstract])) OR (Tumor, Breast[Title/Abstract])) OR (Tumors, Breast[Title/Abstract])) OR (Neoplasms, Breast[Title/Abstract])) OR (Breast Cancer[Title/Abstract])) OR (Cancer, Breast[Title/Abstract])) OR (Mammary Cancer[Title/Abstract])) OR (Cancer, Mammary[Title/Abstract])) OR (Cancers, Mammary[Title/Abstract])) OR (Mammary Cancers[Title/Abstract])) OR (Malignant Neoplasm of Breast[Title/Abstract])) OR (Breast Malignant Neoplasm[Title/ Abstract])) OR (Breast Malignant Neoplasms[Title/Abstract])) OR (Malignant Tumor of Breast[Title/Abstract])) OR (Breast Malignant Tumor[Title/Abstract])) OR (Breast Malignant Tumors[Title/ Abstract])) OR (Cancer of Breast[Title/ Abstract])) OR (Cancer of the Breast[Title/
Abstract])) OR (Mammary Carcinoma, Human[Title/Abstract])) OR (Carcinoma, Human Mammary[Title/Abstract])) OR (Carcinomas, Human Mammary[Title/ Abstract])) OR (Human Mammary Carcinomas[Title/Abstract])) OR (Mammary Carcinomas, Human[Title/Abstract])) OR (Human Mammary Carcinoma[Title/ Abstract])) OR (Mammary Neoplasms, Human [Title/Abstract])) OR (Human Mammary Neoplasm[Title/Abstract])) OR (Human Mammary Neoplasms[Title/ Abstract])) OR (Neoplasm, Human Mammary[Title/Abstract])) OR (Neoplasms, Human Mammary[Title/Abstract])) OR (Mammary Neoplasm, Human[Title/ Abstract])) OR (Breast Carcinoma[Title/ Abstract])) OR (Breast Carcinomas[Title/ Abstract])) OR (Carcinoma, Breast[Title/ Abstract])) OR (Carcinomas, Breast[Title/ Abstract]))).

Participant or population: Breast cancer patients.

Intervention: Single nucleotide polymorphisms associated with breast cancer risk.

Comparator: Healthy person.

Study designs to be included: Case-control study.

Eligibility criteria: Tumor group:1. Patients with breast cancer confirmed by histopathological studies2. There are no restrictions on menopause, gender, age, country or region3. There are no restrictions on breast cancer classification, staging, surgery or drug therapy4. Exclude duplicate reports, animal studies, and review studies; Studies in which SNPs demonstrated a departure from HardyWeinberg equilibrium (HWE) in controls were excluded.

Information sources: Literatures published in Pubmed and Embase database until June 2021 which concern differences in frequency between SNPs of BC patients and normal people were incorporated in our review, with no language limits. Search strategy was following some search terms: 
"SNPs”, “single nucleotide polymorphisms", "Breast cancer", "Breast Neoplasm", etc. Supplementary Materials 1 shows the details regarding the search terms and retrieval type.

Main outcome(s): Studies describing the association between BC risk and SNPs were searched from Pubmed and Embase databases until June 2021. A total of 182 studies $(312,339 \mathrm{BC}$ patients and 358,479 non-cancer controls) included 72 SNPs of 46 genes were included in our metaanalysis process. Firstly, we carried out a paired meta-analysis to calculate the odds ratio (OR) and $95 \%$ confidence intervals (Cls), and then used network meta-analysis and Thakkinstain analys is to comprehensively predict the inherited model of the 14 SNPs screened out, including TNRC9 rs8051542, MTHFR rs1801131, Fas rs2234767, VEGF rs3025039, PHB rs6917, RAD51 rs1801320, TGF $\beta 1$ rs1800469, microRNA rs3746444, IL-8 rs4073, CD44 rs13347, APE1 rs1760944, XRCC1 rs1799782, Leptin rs7799039, BRCA1 rs8176318. The analysis of false positive reporting rate (FPRP) for these 14 SNPs was made. Under the strict criterion of a priori probability value of 0.0001 , the co-dominant model of CD44 rs13347(OR:2.20;95\% CIs:1.67-2.88) showed that the FPRP value was still less than 0.2. It was identified as the best gene for this study. In addition, a candidate gene T NRC 9 rs 8051542 (OR: 1.34; 95\%Cls:1.13-1.59), and two relative dominant genes MTHFR rs1801131, Fas rs2234767 related to risk of $B C$ were also considered.

Quality assessment / Risk of bias analysis: Two reviewers will independently assess risk of bias based on the following domains from recommendations from the Cochrane handbook: 1. Adequate sequence generation; 2.Allocation concealment; 3.Blinding; 4. Incomplete outcome data and how it was addressed; 5. Selective reporting of the outcome.

Strategy of data synthesis: This review used Stata16.0 software to calculate the extracted data. Firstly, pairwise meta-
Analysis was performed to analyze the heterogeneity of different genetic models. The calculated heterogeneity data included value of I square and $P$ value.For the mesh meta-analysis, we used the related software GeMTC (V0.14.3)[4] based on Markov Chain Monte Carlo (MCMC) method. Parallel Markov chain Monte Carlo simulations were run for four times. It contains a burn-in phase of 20,000 stimuli and an additional 50,000 stimuli phase, with a potential scaling factor (PSRF) value equal to 1.0 as the convergence threshold and evaluating the random effects standard deviation. Thakkintain's algorithm was adopted to further analyze the optimal genetic model for the dominant genes selected by network meta-analysis (specific method), In order to judge the reliability of the gene value and compare the final advantages of these genes, we further calculated the false positive reporting rate FPRP, we pre-set an FPRPlevel criterion as $\mathbf{0 . 2}$ for noteworthiness.

Subgroup analysis: We will conduct a subgroup analysis of the SNPs most associated with breast cancer, according to race, whether menopause, age, sex, etc.

Sensitivity analysis: Sensitivity analysis will be conducted to check the robustness and reliability of pooled outcome results.

Country(ies) involved: China.

Keywords: Breast cancer; Case-control study; Genetic model; Network metaanalysis; Thakkinstain algorithm; FPRP.

Contributions of each author:

Author 1 - Ying yuan Wang.

Author 2 - Zechang Chen.

Author 3 - Luxin Zhang.

Author 4 - Shuangyi Chen.

Author 5 - Zhuomiao Ye.

Author 6 - Tingting Xu.

Author 7 - Yingying Zhang.

Conflicts of interest: The authors declare that they have no known competing financial interests or personal relationships that could have appeared to influence the work reported in this paper. 